\title{
Experiência Inicial com o Sistema de Reversão de Fluxo em Pacientes Submetidos a Angioplastia Carotídea
}

\author{
Patrick Bastos Metzger', Samuel Martins Moreira', Nio Mitsuru Izukawa', \\ Fernanda Maria Resegue Angelieri ${ }^{1}$, Marcelo Bueno de Oliveira Colli ${ }^{1}$, Eduardo Rafael Novero ${ }^{1}$, \\ Leandro Cordeiro Soares ${ }^{1}$, Deo Cesar Carneiro Fontes ${ }^{1}$, Eduardo da Silva Jordão', \\ Bruno Lorenção de Almeida', Manoel Nicolas Cano', Ana Claudia Gomes Petisco'1, \\ Mohamed Hassan Saleh', José Eduardo Martins Barbosa', Antonio Massamitsu Kambara ${ }^{1}$
}

\section{RESUMO}

Introdução: O tratamento endovascular da doença aterosclerótica carotídea vem evoluindo continuamente, embora possam ocorrer complicações embólicas ou isquêmicas, mesmo com o uso dos sistemas de proteção cerebral. Este estudo teve como objetivo avaliar os resultados de uma série inicial de pacientes que utilizaram o sistema de reversão de fluxo durante angioplastia carotídea. Métodos: Estudo prospectivo, não-randomizado, não-controlado, realizado em um único centro. Foram incluídos pacientes assintomáticos com lesão $>70 \%$ ou sintomáticos com lesão > 50\% em artéria carótida interna. Foi utilizado o sistema de reversão de fluxo, com pré-dilatação em casos selecionados e uso de stents de células abertas em todos os casos. Avaliou-se a ocorrência de acidentes vasculares encefálicos (AVEs) maiores e menores, ataques isquêmicos transitórios (AITs), infarto agudo do miocárdio (IAM) e óbito até 30 dias pós-procedimento. Resultados: Entre setembro de 2010 e fevereiro de 2011 foram realizadas angioplastias carotídeas em 17 pacientes, a maioria do sexo masculino $(70,6 \%)$, com média de idade de $66,7 \pm 8$ anos, sendo $17,6 \%$ diabéticos. Cerca de metade dos pacientes era sintomática, 5 pacientes tinham história de AVE prévio $(29,4 \%)$ e 3, de AIT $(17,7 \%)$ prévio. Sucesso técnico foi obtido em $100 \%$ dos casos. Houve um caso de óbito $(5,9 \%), 24$ horas após o procedimento, em paciente de alto risco cirúrgico tratado na fase evolutiva de IAM por apresentar AITs de repetição. Não ocorreram casos de AVE maior ou menor ou AIT durante o período de acompanhamento. Conclusões: Neste estudo, o sistema de reversão de fluxo mostrou ser eficiente e seguro em pacientes submetidos a angioplastia carotídea.

DESCRITORES: Estenose das carótidas. Angioplastia. Stents.

\section{ABSTRACT}

Initial Experience with the Flow Reversal System in Patients Undergoing Carotid Angioplasty

Background: The endovascular treatment of carotid atherosclerotic disease has continuously evolved, although ischemic or embolic complications may occur even with the use of cerebral protection systems. This study was aimed at evaluating the perioperative results of an initial series of patients using the flow reversal system during carotid angioplasty. Methods: This was a prospective, non-randomized, non-controlled, singlecenter study. Asymptomatic patients with lesions $>70 \%$ or symptomatic patients with lesions $>50 \%$ in the internal carotid artery were included. The flow reversal system was used with pre-dilation in selected cases and open-cell stents were used in all cases. We evaluated the occurrence of major and minor strokes, transient ischemic attacks (TIAs), acute myocardial infarction (AMI) and death within 30 days after the procedure. Results: Between September 2010 and February 2011 carotid angioplasty was performed in 17 patients, most of them male $(70.6 \%)$, with mean age of $66.7 \pm$ 8 years, of which $17.6 \%$ were diabetic. Approximately half of the patients were symptomatic, 5 patients had a prior history of stroke (29.4\%) and 3 had TIA (17.7\%). Technical success was achieved in $100 \%$ of the patients. There was one death $(5.9 \%), 24$ hours after the procedure in a high surgical risk patient treated in the evolution phase of an AMI due to repetitive TIAs. There were no cases of major or minor strokes or TIAs during the follow-up. Conclusions: In our study, the flow reversal system proved to be effective and safe in patients undergoing carotid angioplasty.

KEY-WORDS: Carotid stenosis. Angioplasty. Stents.

1 Instituto Dante Pazzanese de Cardiologia - São Paulo, SP, Brasil. Correspondência: Patrick Bastos Metzger. Av. Dr. Dante Pazzanese, 500 - Vila Mariana - São Paulo, SP, Brasil - CEP 04012-180 E-mail: patrickvascular@gmail.com

Recebido em: 2/4/2011 • Aceito em: 8/6/2011 
A doença cerebrovascular é uma das principais causas de morte e incapacidade em nosso País. Estima-se que ocorram cerca de 250 mil acidentes vasculares encefálicos (AVEs) por ano no Brasil, dos quais cerca de $85 \%$ são de etiologia isquêmica. ${ }^{1}$ De acordo com dados epidemiológicos, cerca de cem mil pessoas por ano poderiam se beneficiar profilaticamente do tratamento de suas lesões carotídeas, evitando-se as sequelas de um AVE, mas somente a minoria é privilegiada. ${ }^{2,3} \mathrm{O}$ tratamento endovascular da doença aterosclerótica carotídea vem evoluindo continuamente com o advento de novos stents e novos mecanismos de proteção cerebral. A maior limitação para angioplastia carotídea é o risco potencial de eventos neurológicos catastróficos associados ao procedimento. ${ }^{4} \mathrm{~A}$ embolização cerebral é uma ocorrência universal durante os procedimentos de angioplastia carotídea, bem documentada por diversos estudos ex vivo. ${ }^{5-7}$ A finalidade dos sistemas de proteção cerebral, por dispositivos oclusores ou filtros, durante a angioplastia carotídea é suprimir ou diminuir o número de partículas liberadas na circulação cerebral durante o procedimento. ${ }^{2}$

O objetivo deste estudo foi avaliar os resultados de uma série inicial de pacientes que utilizaram o sistema de proteção cerebral por reversão de fluxo durante angioplastia carotídea.

\section{MÉTODOS}

Trata-se de um estudo prospectivo, não-randomizado, não-controlado, realizado em um único centro, entre setembro de 2010 e fevereiro de 2011. Foram incluídos no estudo pacientes de ambos os sexos, com idade $>18$ anos e $<80$ anos, assintomáticos com lesão $>70 \%$ ou sintomáticos com lesão > 50\% em artéria carótida interna. Foram considerados sintomáticos os pacientes com antecedente de ataque isquêmico transitório (AIT), amaurose fugaz ou AVE no hemisfério suprido pelo vaso-alvo nos últimos seis meses pré-procedimento. Foram excluídos os pacientes que apresentaram insuficiência renal crônica não-dialítica (creatinina $>2 \mathrm{mg} / \mathrm{dl}$ ), alergia ao uso de contraste iodado, lesão oclusiva da carótida externa contralateral, lesão obstrutiva significativa ou oclusiva da carótida externa ipsilateral, hemorragia cerebral $<1$ mês. O diagnóstico da gravidade da estenose foi baseado no ultrassom com Doppler de carótidas e confirmado com a realização de angiografia cerebral. Nos pacientes sintomáticos foi realizada tomografia cerebral prévia ao procedimento, para documentação da lesão neurológica.

Após a angioplastia carotídea, os pacientes realizaram novo ultrassom com Doppler antes da alta hospitalar com a finalidade de avaliar a perviedade do stent e a presença de estenoses residuais com repercussão hemodinâmica.

Todos os pacientes utilizaram aspirina na dose de $200 \mathrm{mg} /$ dia e clopidogrel na dose de $75 \mathrm{mg} / \mathrm{dia}$, iniciados pelo menos três dias antes do procedimento.

\section{Sistema de proteção cerebral}

Foi utilizado para proteção cerebral o Sistema de Reversão de Fluxo (Gore Medical, Flagstaff, Estados Unidos), que consiste de três componentes: um cateter com um balão oclusor localizado em sua extremidade distal, um balão montado em um guia, e um filtro externo (Figura 1). A luz do cateter permite a passagem de balões e stents. O cateter é posicionado na carótida comum e o guia, na carótida externa. O sistema é conectado ao cateter inserido na veia femoral contralateral, para criar um shunt arteriovenoso temporário. São insuflados o balão na carótida externa, ocluindo o fluxo, e, em seguida, o balão na carótida comum, criando um fluxo retrógrado na carótida interna. Durante a angioplastia carotídea, as partículas geradas no procedimento são capturadas em um filtro externo, interposto entre os cateteres da artéria e veia femorais.

\section{Angioplastia carotídea}

A angioplastia carotídea foi realizada sob anestesia local, com paciente em decúbito dorsal, no laboratório de hemodinâmica. As punções percutâneas da artéria femoral ipsilateral e da veia femoral contralateral foram realizadas pela técnica de Judkins. Foram inseridos um introdutor $9 \mathrm{~F}$ em artéria femoral comum e um introdutor $6 \mathrm{~F}$ em veia femoral comum, em sentido cranial. A cateterização do óstio da artéria carótida comum foi obtida com cateter-guia JR ou Simmons curva 2, precedido por um guia hidrofílico 0,035 polegada x $160 \mathrm{~cm}$. Procedeu-se, em seguida, à troca do fio-guia hidrofílico por um fio-guia extrassuporte 0,035 polegada $x$ $260 \mathrm{~cm}$. O cateter-guia foi, então, trocado pelo cateter SRF, posicionado na artéria carótida comum. Nesse momento, foram administradas 7.500 unidades de heparina não-fracionada por via endovenosa e o balão distal foi posicionado na artéria carótida externa. O filtro externo foi conectado aos cateteres femorais, arterial e venoso. O balão distal foi insuflado na carótida externa e, em seguida, o balão proximal, na artéria carótida comum. Com a abertura do shunt arteriovenoso estabeleceu-se a reversão do fluxo cerebral pela carótida interna, confirmada pela angiografia de controle. A pré-dilatação foi realizada somente nas lesões suboclusivas, a prótese utilizada foi o stent de nitinol PRECISE $^{\mathrm{TM}}$ (Cordis Corporation, Bridgewater, Estados Unidos) $8 \mathrm{~mm}$ x $40 \mathrm{~mm}$, e a pós-dilatação foi obtida com um balão de baixo perfil 0,014 polegada, de troca rápida, com diâmetro de $5,5 \mathrm{~mm}$ ou $6 \mathrm{~mm}$ e extensão de $20 \mathrm{~mm}$. Angiografia de controle final das carótidas e circulação cerebral foi realizada após a desinsuflação dos balões proximal e distal (Figura 2).

\section{Desfechos e definições}

Foram avaliados o sucesso técnico do procedimento, a ocorrência de AVE maior ou menor, AIT, infarto agudo do miocárdio (IAM) e óbito até 30 dias após o procedimento. Os eventos neurológicos foram 


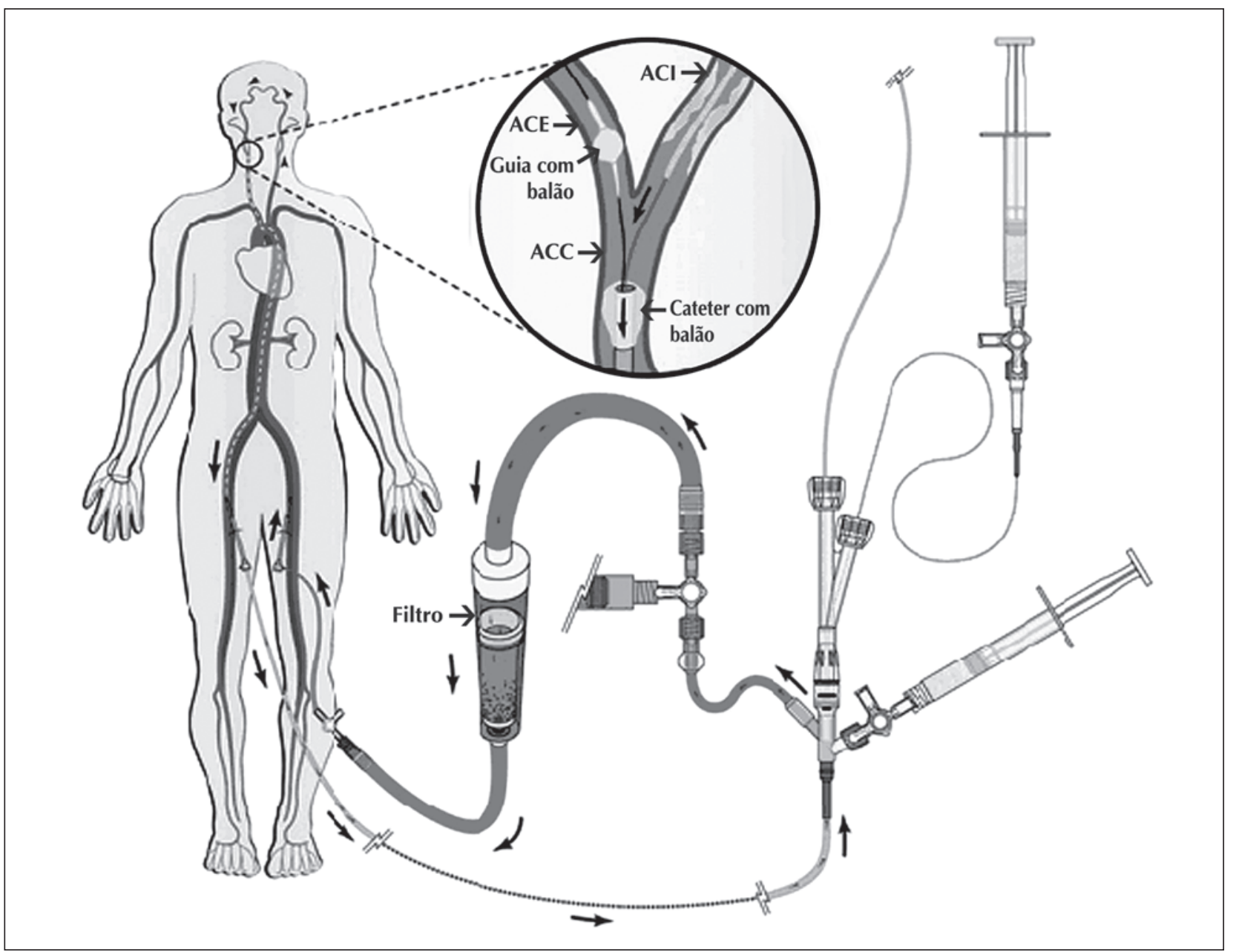

Figura 1 - Representação esquemática* demonstrando o funcionamento do Sistema de Reversão de Fluxo (Gore Medical, Flagstaff, Estados Unidos). $\mathrm{ACC}=$ artéria carótida comum; $\mathrm{ACE}=$ artéria carótida externa; $\mathrm{ACl}=$ artéria carótida interna.

definidos como: 1. AVE maior - déficit neurológico estabelecido, incapacitante ou que levou a óbito; 2. AVE menor - déficit neurológico estabelecido, não-incapacitante ou com sequelas neurológicas mínimas; e 3. AIT - déficit neurológico reversível $<24$ horas.

\section{RESULTADOS}

Foram realizadas angioplastias carotídeas em 17 pacientes, a maioria era do sexo masculino $(70,6 \%)$, com média de idade de $66,7 \pm 8$ anos (variação de 50-78 anos), e 17,6\% eram diabéticos. Cerca de 50\% dos pacientes eram sintomáticos, 5 tinham história de AVE $(29,4 \%)$ e 3 de AIT $(17,7 \%)$ prévios. Cinco pacientes $(29,4 \%)$ foram considerados de alto risco cirúrgico, com escore de risco da American Society of Anestesiology > 3. Quatro pacientes (23,5\%) apresentavam quadro angiográfico de maior gravidade, com lesões críticas bilaterais (Tabela). Foram realizadas angioplastias carotídeas em todos os casos, obtendo-se sucesso técnico de $100 \%$. Em 2 casos não foi possível a insuflação do balão da artéria carótida externa (um caso por dificuldade técnica no acesso e outro por falha do material), porém foram conseguidas a reversão de fluxo e a conclusão do procedimento. Houve 1 caso de óbito $(5,9 \%)$ que ocorreu 24 horas após o procedimento em paciente de alto risco cirúrgico, tratado na fase evolutiva de IAM por apresentar AITs de repetição. Apresentava má função ventricular (fração de ejeção 29\%), não tendo sido identificada fonte emboligênica intracardíaca. Não ocorreram casos de AVE maior ou menor ou AIT durante o período de acompanhamento. Ocorreram 2 casos de hipotensão associados a bradicardia, os quais foram responsivos à expansão da volemia e ao uso de atropina, sem resultar em comprometimento neurológico. Não houve

* Obtida em: http//:geromedical.com/flowreversal/ 

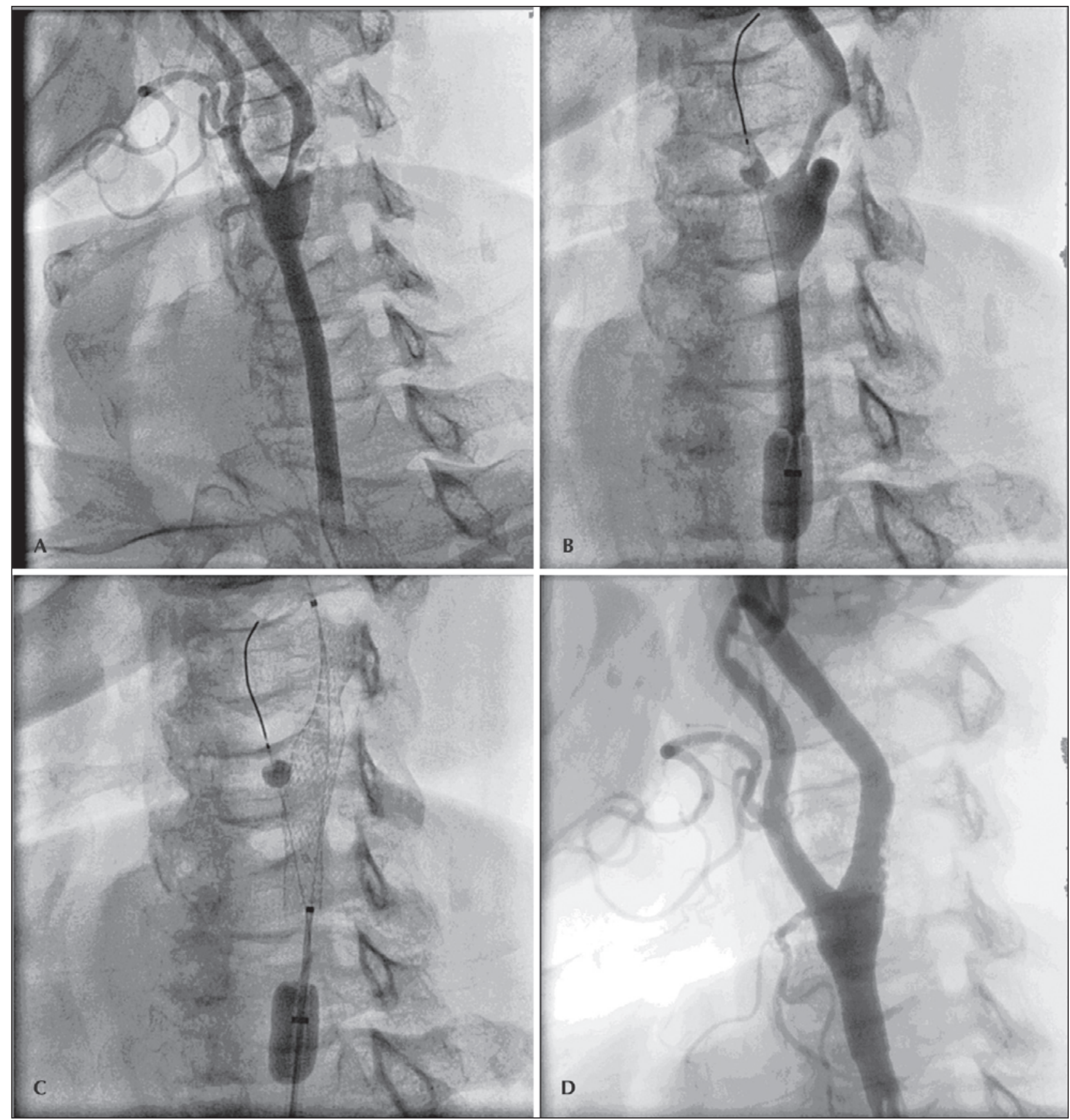

Figura 2 - Angioplastia carotídea. Em A, estenose crítica em artéria carótida interna. Em B, balões insuflados em artérias carótidas externa e comum. Em C, implante de stent em artéria carótida interna. Em D, resultado angiográfico final após dilatação com balão.

casos de intolerância neurológica durante a insuflação dos balões para o estabelecimento da reversão do fluxo ou sangramentos. Dezesseis $(94,1 \%$ ) pacientes tiveram alta hospitalar 2 dias após a internação, sem intercorrências clínicas ou neurológicas durante o período intra-hospitalar e com ultrassom com Doppler de carótidas realizado no primeiro dia de pós-operatório demonstrando perviedade do stent e sem lesões residuais com repercussão hemodinâmica. Não ocorre- ram eventos clínicos adicionais nos primeiros 30 dias em qualquer dos pacientes tratados.

\section{DISCUSSÃO}

A complicação mais temida durante a angioplastia carotídea é a embolização cerebral. Esta tem sua maior incidência durante o período periprocedimento, quando as partículas que se desprendem durante o procedi- 
TABELA

Dados clínicos e angiográficos

\begin{tabular}{lc}
\hline Características da população & $\mathbf{n}=\mathbf{1 7}$ \\
\hline Média de idade, anos & $66,7 \pm 8$ \\
Sexo, n (\%) & $12(70,6)$ \\
$\quad$ Masculino & $13(76,5)$ \\
Tabagismo, n (\%) & $3(17,6)$ \\
Diabetes melito, n (\%) & $17(100)$ \\
Hipertensão, n (\%) & $15(88,2)$ \\
Dislipidemia, n (\%) & $5(29,4)$ \\
Infarto do miocárdio prévio, n (\%) & $3(17,6)$ \\
Revascularização do miocárdio, n (\%) & $8(47,1)$ \\
Doença sintomática, n (\%) & $5(29,4)$ \\
AVE & $1(5,9)$ \\
AIT & $2(11,8)$ \\
AIT retiniano & $5(29,4)$ \\
Escore ASA > 3 & $4(23,5)$ \\
Lesão em ACl contralateral (> 70\%), n (\%)
\end{tabular}

$\mathrm{ACl}=$ artéria carótida interna; $\mathrm{AIT}=$ ataque isquêmico transitório prévio; ASA = American Society of Anestesiology; $\mathrm{AVE}=$ acidente vascular encefálico prévio; $\mathrm{n}=$ número de pacientes.

mento podem ou não gerar déficits neurológicos, de acordo com seu tamanho. Êmbolos com menos de $20 \mu \mathrm{m}$ atravessam livremente a circulação cerebral, os que têm entre $20 \mu \mathrm{m}$ e $200 \mu \mathrm{m}$ podem ocasionar microinfartos cerebrais, e os com mais de $200 \mu \mathrm{m}$ causam danos cerebrais significativos. ${ }^{8}$ Atualmente, o sistema de proteção cerebral durante as angioplastias carotídeas é requisito fundamental para o procedimento. A taxa de eventos cerebrais isquêmicos e de mortalidade é de $5,3 \%$ sem o uso dos sistemas de proteção cerebral e de $2,3 \%$ com sua utilização. ${ }^{9}$ O primeiro sistema de proteção cerebral desenvolvido foi o sistema oclusor por balão na artéria carótida interna, na artéria carótida comum ou em ambas, sendo modificado posteriormente (técnica de Karchel). ${ }^{10}$ Apesar de terem baixo perfil e de evitarem as embolizações cerebrais, esses sistemas interrompiam o fluxo carotídeo completamente, além de cruzar o segmento estenótico sem que o sistema estivesse montado, podendo levar a eventos cerebrais isquêmicos nessa fase do procedimento. ${ }^{8}$ Esse tipo de proteção cerebral pode não ser bem tolerado nos pacientes com circulação inadequada pelo polígono de Willis ou com graus de estenoses contralaterais graves, podendo ocorrer em até 10\% dos casos tratados. ${ }^{11}$ Os filtros foram o segundo sistema de proteção desenvolvido e são compostos de uma membrana polimérica com poros com menos de $200 \mu \mathrm{m}$. Diversos estudos relatam que esse sistema de proteção cerebral pode capturar mais de $90 \%$ das partículas embolizadas durante a angioplastia carotídea. ${ }^{7}$ Tem como vantagens seu fácil manuseio, assim como mantém o fluxo cerebral durante todo seu procedimento. Apresentam como desvantagens a necessidade de cruzar a lesão para o posicionamento do sistema de proteção, assim como a possibilidade de causar danos à artéria, com espasmos arteriais e dissecções. Esse sistema exige um segmento arterial sem tortuosidades ou placas, para que seja utilizado. Apesar de tudo, ainda é possível a ocorrência de embolizações cerebrais com esse sistema, comprovadas por estudos com a utilização do ultrassom com Doppler transcraniano e ressonância nuclear magnética por difusão. ${ }^{12,13} \mathrm{O}$ novo sistema de proteção cerebral por reversão de fluxo apresenta como vantagens, em relação aos outros sistemas: a inversão do fluxo da artéria carótida interna diretamente para o sistema venoso, via veia femoral, minimizando a possibilidade de embolizações cerebrais durante o procedimento carotídeo; a manutenção da perfusão cerebral durante todo o procedimento, desde que o sistema de colaterais esteja pérvio; e o fato de não haver manipulação da placa carotídea durante a implantação do sistema. ${ }^{8}$

As contraindicações à utilização da proteção cerebral por reversão de fluxo são a obstrução do sistema de artérias comunicantes cerebrais e a intolerância neurológica durante o período de reversão do fluxo intracerebral. A oclusão da artéria carótida externa ipsilateral à carótida interna a ser tratada é uma contraindicação relativa, uma vez que a reversão de fluxo cerebral pode ser conseguida apenas com a oclusão da artéria carótida comum na maioria dos casos. Estudos clínicos utilizando a angioplastia carotídea com sistemas de proteção cerebral em pacientes com alto risco demonstraram taxas de AVE nos 30 dias de acompanhamento de 3,8\% e 5,3\% nos estudos SAPPHIRE e ARCHeR, respectivamente. ${ }^{14,15}$ Neste estudo, observou-se 1 óbito em um paciente com IAM recente, com má função ventricular e AITs de repetição durante a internação hospitalar. Observou-se que nos pacientes avaliados não houve desfechos neurológicos isquêmicos e as complicações intraprocedimento foram revertidas com tratamento adequado, não resultando em déficits neurológicos. O ensaio clínico realizado por Parodi et al. ${ }^{16}$ avaliou 46 pacientes submetidos a angioplastia carotídea com e sem proteção cerebral. Desses pacientes, 25 receberam filtros $(n=6)$, balões oclusores $(n=10)$ e sistemas de reversão de fluxo $(n=9)$ e foram comparados a 21 pacientes sem proteção cerebral. Dois eventos menores (AIT e AVE menor) ocorreram no grupo controle. Não ocorreram óbitos, AVEs maiores ou lesões de nervos cranianos. Nesse estudo foi avaliada a quantidade de partículas cerebrais embolizadas durante o procedimento com o uso do ecocardiografia Doppler transcraniana, sendo observado menor número de embolizações cerebrais durante a realização de angioplastia carotídea com uso de proteção cerebral por reversão de fluxo, porém sem repercussão clínica. 
O sistema de proteção cerebral por reversão de fluxo apresenta como desvantagens: seu complexo manuseio, tornando a curva de aprendizado para a utilização do dispositivo mais longa; a necessidade de utilização de dois acessos vasculares (uma punção venosa e uma punção arterial) com introdutor de maior perfil; a grande extensão do shunt arteriovenoso, que, pelo maior espaço morto existente, pode propiciar, quando indevidamente manipulado, estase sanguínea e trombose no sistema.

Existem etapas durante a angioplastia carotídea em que o cérebro não está protegido: quando é obtida a angiografia do arco aórtico, durante a canulação das artérias, e durante o avanço de fios-guia e cateteres na artéria carótida comum. Isso ocorre com todos os sistemas de proteção cerebral, inclusive com o sistema de reversão de fluxo. Por isso, a presença de morfologia desfavorável e calcificação do arco aórtico, assim como de tortuosidades das artérias carótidas, está relacionada a complicações desse procedimento, independentemente do tipo de proteção cerebral escolhido. ${ }^{17,18}$ Mesmo durante a utilização do sistema de proteção cerebral, a realização de múltiplas trocas de fios e cateteres-guia, a utilização de cateteres liberadores do stent, assim como a pós-dilatação do stent realizada com os mais diversos balões geram riscos de eventos embólicos cerebrais, seja por micropartículas seja por bolhas de ar.

\section{CONCLUSÕES}

O sistema de proteção cerebral por reversão de fluxo demonstrou, neste estudo, ser um sistema eficiente e fácil de utilizar nos pacientes que serão submetidos a angioplastia carotídea. O aperfeiçoamento contínuo dos sistemas de proteção cerebral tem permitido que o tratamento endovascular da doença carotídea seja realizado de maneira cada vez mais segura e efetiva.

\section{CONFLITO DE INTERESSES}

Samuel M. Moreira e Antonio M. Kambara são preceptores e orientadores de instalação de dispositivos endovasculares da empresa Gore Medical (Flagstaff, Estados Unidos). Os demais autores e coautores declaram não haver conflito de interesses relacionado a este manuscrito.

\section{REFERÊNCIAS}

1. Lessa I. Epidemiologia das doenças cerebrovasculares no Brasil. Rev Soc Cardiol Estado de São Paulo. 1999;9(4):509-18.

2. Ristow AV. The ups and downs in the treatment of carotid disease [editorial]. J Vasc Bras. 2007;6(4):303-6.
3. Bonamigo TP, Lucas ML. Análise crítica das indicações e resultados do tratamento cirúrgico da doença carotídea. J Vasc Bras. 2007;6(4):366-77.

4. Théron J, Raymond J, Casasco A, Courtheoux F. Percutaneous angioplasty of atherosclerotic and postsurgical stenosis of carotid arteries. AJNR Am J Neuroradiol. 1987;8(3):495-500.

5. Kastrup A, Schulz JB, Raygrotzki S, Gröschel K, Ernemann U. Comparison of angioplasty and stenting with cerebral protection versus endarterectomy for treatment of internal carotid artery stenosis in elderly patients. J Vasc Surg. 2004;40(5):945-51.

6. Lam RC, Lin SC, DeRubertis B, Hynecek R, Kent KC, Faries PL. The impact of increasing age on anatomic factors affecting carotid angioplasty and stenting. J Vasc Surg. 2007;45(5): 875-80.

7. MacDonald S. Is there any evidence that cerebral protection is beneficial? Experimental data. J Cardiovasc Surg. 2006; 47(2):127-36.

8. Kambara AM, Cano NM, Moreira SM, Novero ER. Angioplatia carotídea: donde estamos y hacia dónde vamos. Bol Proeducar. 2010;(6):7-10.

9. Wholey $\mathrm{MH}, \mathrm{Al}-$ Mubarek $\mathrm{N}$, Wholey $\mathrm{MH}$. Updated review of the global carotid artery stent registry. Catheter Cardiovasc Interv. 2003;60(2):259-66.

10. Ohki T, Roubin G, Veith FJ, Iyer SS, Brady E. Efficacy of a filter device in the prevention of embolic events during carotid angioplasty and stenting: an ex vivo analysis. J Vasc Surg. 1999;30(6):1034-44.

11. Wain RA, Veith FJ, Berkowitz BA, Legatt AD, Schwartz M, Lipsitz EC, et al. Angiographic criteria reliably predict when carotid endarterectomy can be safely performed without a shunt. J Am Coll Surg. 1999;189(1):93-100.

12. Schmidt A, Diederich KW, Scheinert S, Braunlich S, Olenburger $\mathrm{T}$, Biamino G, et al. Effect of two different neuroprotection systems on microembolization during carotid artery stenting. J Am Coll Cardiol. 2004;44(10):1966-9.

13. Gattuso R, Martinelli O, Alunno A, D'Angeli I, Felli M, Castiglione $\mathrm{A}$, et al. Carotid stenting and transcranial Doppler monitoring: indications for carotid stenosis treatment. Vasc Endovascular Surg. 2010;44(7):535-8.

14. Schonholz CJ, Uflacker R, Parodi JC, Hannegan C, Selby B. Is there evidence that cerebral protection is beneficial? Clinical data. J Cardiovasc Surg (Torino). 2006;47(2):137-41.

15. Gray WA, Hopkins LN, Yadav S, Davis T, Wholey M, Atkinson $R$, et al.; ARCHeR Trial Collaborators. Protected carotid stenting in hight-surgical-risk patients: the ARCHeR results. J Vasc Surg. 2006;44(2):258-68. Erratum in: J Vasc Surg. 2007;45(1):226.

16. Parodi JC, La Mura R, Ferreira LM, Mendez MV, Cersósimo $\mathrm{H}$, Schönholz C, et al. Initial evaluation of carotid angioplasty and stenting with three different cerebral protection devices. J Vasc Surg. 2000;32(6):1127-36.

17. Stingele R, Berger J, Alfke K, Eckstein HH, Fraedrich G, Allenberg J, et al.; SPACE Investigators. Clinical and angiographic risk factors for stroke and death within 30 days after carotid endarterectomy and stent-protected angioplasty: a subanalysis of the SPACE study. Lancet Neurol. 2008;7(3):216-22.

18. Massiére B, Ristow AV, Vieira RD, Cury JM, Gress M, Vescovi $A$, et al. Carotid angioplasty with flow reversion in octogenarians: a case report. J Vasc Bras. 2010;9(3):186-9. 